\title{
Oncologic and Anastomotic Safety of Low Ligation of the Inferior Mesenteric Artery With Additional Lymph Node Retrieval: A Case-Control Study
}

\author{
Cho Shin Kim, Sohyun Kim \\ Department of Surgery, Yeungnam University College of Medicine, Daegu, Korea
}

Purpose: We assessed the oncologic and anastomotic benefits of low ligation of the inferior mesenteric artery (IMA) with additional lymph node (LN) retrieval.

Methods: We performed a retrospective case-control study between January 2011 and July 2015. All patients underwent curative resection of a primary sigmoid or rectal tumor. We excluded patients with distant metastases at the time of diagnosis. The case group included patients who underwent high ligation of the IMA (high group, HG). The control group included patients who underwent low ligation of the IMA with low group with additional LN retrieval (LGAL). Controls were identified by matching patients based on age ( \pm 5 years), sex, tumor location, and final histopathological stage. Finally, each group included 97 patients.

Results: Clinical characteristics did not significantly differ between groups. The mean number of additional harvested LN was 2.19 (range, $0-11$ ), and one patient in the LGAL had a metastatic LN among the additional harvested LN. The overall morbidity was $22.7 \%$ in the HG and $30 \%$ in the LGAL $(\mathrm{P}=0.257)$. Anastomotic leakage occurred in 14 patients $(14.4 \%)$ in the HG and 5 patients $(5.2 \%)$ in the LGAL $(\mathrm{P}=0.030)$. The mean disease-free survival time in the HG was longer than that in the LGAL $(\mathrm{P}=0.008)$. The mean overall survival $(\mathrm{OS})$ time was $70.4 \pm 1.3$ months. The mean OS was $63.7 \pm 1.6$ months in the HG and $69.1 \pm 2.6$ months in the LGAL $(\mathrm{P}=0.386)$.

Conclusion: Low ligation of the IMA with additional LN retrieval is technically safe. However, the oncologic effect was better after high ligation of IMA.

Keywords: Inferior mesenteric artery; High ligation; Low ligation; Colorectal neoplasms

\section{INTRODUCTION}

Ligation of the inferior mesenteric artery (IMA) is essential for curative resection of colorectal cancer. Adequate ligation of the IMA ensures en bloc excision of lymph nodes (LN) associated with a tumor [1]. The ligation techniques routinely used globally

Received: August 25, 2018 - Accepted: October 9, 2018

Correspondence to: Sohyun Kim, M.D.

Department of Surgery, Yeungnam University Medical Center, Yeungnam University College of Medicine, 170 Hyeonchung-ro, Nam-gu, Daegu 42415, Korea

Tel: +82-53-620-3594, Fax: +82-53-624-1213, E-mail: kshgs@yu.ac.kr ORCID code: https://orcid.org/0000-0002-8625-329X

(C) 2019 The Korean Society of Coloproctology

This is an open-access article distributed under the terms of the Creative Commons Attribution NonCommercial License (http://creativecommons.org/licenses/by-nc/4.0) which permits unrestricted noncommercial use, distribution, and reproduction in any medium, provided the original work is properly cited. are ligation of the IMA at its origin from the aorta (high ligation) or ligation of the IMA below the origin of the left colic artery (low ligation) $[1,2]$. High ligation of the IMA is a universally accepted technique $[2,3]$. However, the appropriate level of IMA ligation remains controversial [4].

The level of IMA ligation (both, high, and low) is associated with specific advantages and disadvantages. High ligation of the IMA preserves an adequate length of the colon to perform a successful anastomosis [5]. A few studies have shown that high ligation provides a longer colonic segment than that available with low ligation [5, 6]. High ligation of the IMA facilitates apical LN dissection [7]. However, the risk of ischemic colitis or ischemic complications is higher in patients undergoing high ligation of the IMA [8]. Despite the oncologic advantages of high over low ligation, previous studies have failed to demonstrate better survival rates with the former technique $[1,7,9]$. Low ligation of the IMA 
is less invasive and offers the advantage of maintaining adequate anastomotic perfusion [10].

Oncologic, anatomical, and technical factors determine the level of IMA ligation [2]. Recently, the occurrence of colorectal cancer has increased in elderly patients, who often present with several comorbidities [11]. Thus, low ligation of the IMA is preferred in this patient population secondary to the safety and other advantages of the procedure. However, the oncologic benefits of high ligation cannot be ignored. We hypothesized that the oncologic benefit of low ligation of the IMA with additional LN retrieval at its origin is similar to that associated with high ligation. In this study, we aimed to assess the oncologic benefits of low ligation of the IMA with additional LN retrieval at its origin.

\section{METHODS}

A retrospective review was performed of a prospectively collected consecutive series between January 2011 and July 2015. All patients underwent curative resection of a primary colorectal tumour. The inclusion criteria were (1) histologically proven adenocarcinoma; (2) tumor location in the sigmoid colon or rectum; and (3) curative resection with IMA ligation. The exclusion criteria were (1) distant metastasis at the time of diagnosis; (2) synchronous colorectal cancer; (3) metachronous colorectal cancer; (4) other malignancy; (5) diagnosis of familial adenomatous polyposis; and (6) a follow-up period less than 24 months. We reviewed the medical records of 1,115 patients. Of them, 507 matched inclusion and exclusion criteria (Fig. 1). This study was approved by the Institutional Review Board of Yeungnam University Medical Center (approval number: 2018-07-040). The Inter-

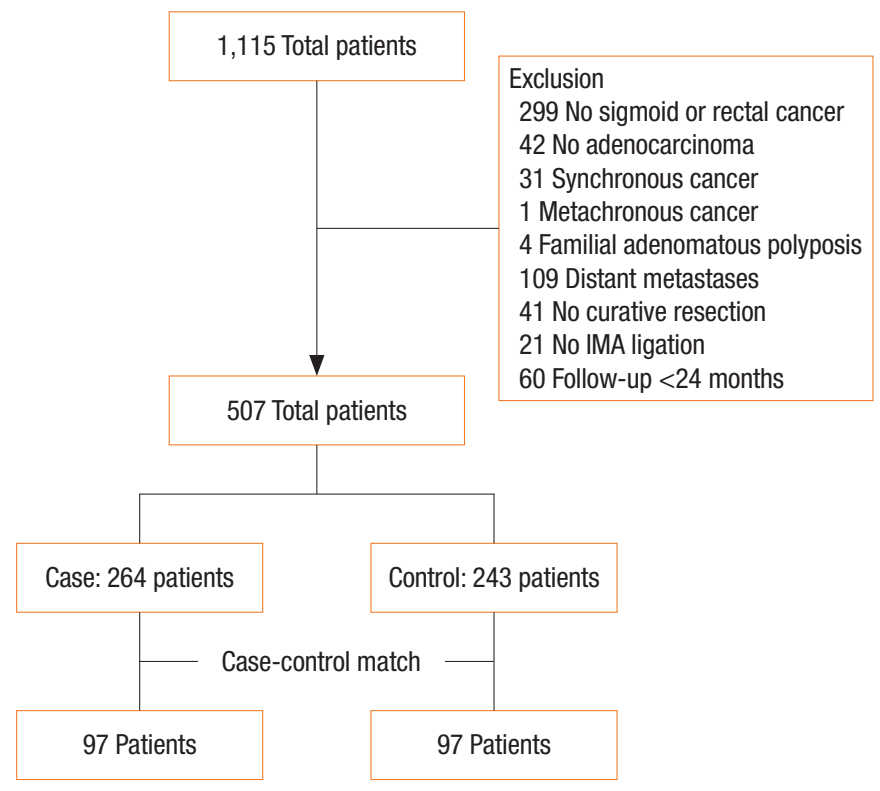

Fig. 1. Study diagram. IMA, inferior mesenteric artery. nal Review Board exempted it from obtaining informed consent from the patients.

\section{Cases and controls}

A case was defined as a patient who underwent high ligation of the IMA (high group, HG). A control was defined as a patient who underwent low ligation of the IMA with additional LN retrieval (low group with additional LN retrieval, LGAL). Controls were identified by matching patients based on age ( \pm 5 years), sex, tumor location, and final histopathological tumor stage. Therefore, one control individually matched for age, sex, tumor location, and final histopathological stage was selected per case. Finally, each group included 97 patients.

\section{IMA ligation and additional $\mathrm{LN}$ retrieval}

All operations were performed by 2 senior surgeons. Laparoscopic resection was performed using a standard medial-to-lateral approach. Dissection was performed using a no-touch isolation technique with early dissection of vessels. Mobilization of the splenic flexure was performed if needed. Rectal surgery included partial or total removal of the mesorectum circumferentially with preservation of the hypogastric nerve and the pelvic plexus. Open resection was performed based on standard surgical procedures. Pre- and postoperative procedures were similar for open and laparoscopic resections.

The inferior mesenteric LNs are located around the origin of the IMA. High ligation was defined as ligation of the IMA at its root with LN removal. Low ligation was defined as ligation of the IMA below the origin of the left colic artery. All patients undergoing low ligation underwent additional LN retrieval, which was defined as removal of LN between the root of the IMA and ligation of the IMA.

\section{Adjuvant therapy}

Some patients with rectal cancer received preoperative chemoradiotherapy. The patients presented with clinically $\mathrm{T} 3$ or $\mathrm{T} 4$ disease or positive nodes. The extent of tumor was evaluated by endorectal ultrasonography or magnetic resonance imaging. Patients received conventional schemes of 40 to 50 Gy delivered over 4 to 5 weeks. Some patients received fluorouracil (FU) and leucovorin (LV) during the first and fifth weeks of pelvic radiotherapy. Others received capecitabine during pelvic radiotherapy. Patients underwent surgery 6 to 8 weeks after preoperative chemoradiotherapy.

Patients with pathologic stage III disease received adjuvant chemotherapy with the FOLFOX regimen (oxaliplatin, FU, LV) or capecitabine. Some patients with pathologic T3 or T4 rectal cancer or positive nodes underwent postoperative chemoradiotherapy based on the same preoperative chemoradiotherapy schedule.

\section{Follow-up}

Short-term outcome after curative resection was 30-day postoperative complications. Anastomotic leakage was defined as a de- 
Table 1. Clinicopathologic characteristics

\begin{tabular}{|c|c|c|c|}
\hline Characteristic & $H G(n=97)$ & $\operatorname{LGAL}(n=97)$ & P-value \\
\hline Sex & & & 1.000 \\
\hline Female & $31(32.0)$ & $31(32.0)$ & \\
\hline Male & $66(68.0)$ & $66(68.0)$ & \\
\hline Age (yr) & $64.9 \pm 9.7$ & $65.1 \pm 9.4$ & 0.898 \\
\hline Preoperative CEA & $3.2(0.6-50.8)$ & $3.8(0.5-287.5)$ & 0.114 \\
\hline ASA PS classification & & & 0.695 \\
\hline $1-\|$ & $83(85.6)$ & $80(82.5)$ & \\
\hline III-IV & $14(14.4)$ & $17(17.5)$ & \\
\hline Tumor location & & & 1.000 \\
\hline Rectum & $45(46.4)$ & $45(46.4)$ & \\
\hline Sigmoid & $52(53.6)$ & $52(53.6)$ & \\
\hline Preoperative chemoradiotherapy & & & 0.566 \\
\hline No & $89(91.8)$ & $92(94.8)$ & \\
\hline Yes & $8(8.2)$ & $5(5.2)$ & \\
\hline Operation & & & 0.086 \\
\hline Laparoscopic & 81 (83.5) & 77 (79.4) & \\
\hline Open & $11(11.3)$ & 19 (19.6) & \\
\hline Robot & $5(5.2)$ & $1(1.0)$ & \\
\hline Operation time (min) & $185.3 \pm 58.1$ & $170.8 \pm 51.4$ & 0.067 \\
\hline Cell differentiation & & & 0.083 \\
\hline WD & $10(10.3)$ & $16(16.5)$ & \\
\hline MD & $79(81.4)$ & $79(81.4)$ & \\
\hline PD/MU/SRC & $8(8.2)$ & $2(2.1)$ & \\
\hline Lymphatic invasion & & & 0.593 \\
\hline No & $64(66.0)$ & $62(63.9)$ & \\
\hline Yes & $33(34.0)$ & $34(35.1)$ & \\
\hline Indeterminate & $0(0)$ & $1(1.0)$ & \\
\hline Vascular invasion & & & 0.591 \\
\hline No & $87(89.7)$ & $87(89.7)$ & \\
\hline Yes & $10(10.3)$ & $9(9.3)$ & \\
\hline Indeterminate & $0(0)$ & $1(1.0)$ & \\
\hline Neural invasion & & & 0.827 \\
\hline No & $64(66.0)$ & $62(63.9)$ & \\
\hline Yes & $32(33.0)$ & $33(34.0)$ & \\
\hline Indeterminate & $1(1.0)$ & $2(2.1)$ & \\
\hline Postoperative radiotherapy & & & 0.368 \\
\hline No & $96(99.0)$ & $93(95.9)$ & \\
\hline Yes & $1(1.0)$ & $4(4.1)$ & \\
\hline Adjuvant chemotherapy & & & 0.886 \\
\hline No & 47 (48.5) & 48 (49.5) & \\
\hline Yes & $50(51.5)$ & $49(50.5)$ & \\
\hline
\end{tabular}

(Continued to the next)
Table 1. Continued

\begin{tabular}{lccc}
\hline Characteristic & HG $(n=97)$ & LGAL $(n=97)$ & P-value \\
\hline Pathologic stage & & & 1.000 \\
I & $23(23.7)$ & $23(23.7)$ & \\
IIA & $32(33.0)$ & $32(33.0)$ & \\
IIB & $1(1.0)$ & $1(1.0)$ & \\
IIIA & $5(5.2)$ & $5(5.2)$ & \\
IIIB & $30(30.9)$ & $30(30.9)$ & \\
IIIC & $6(6.2)$ & $6(6.2)$ & \\
\hline
\end{tabular}

Values are presented as number (\%), mean \pm standard deviation, and median (range).

HG, high group; LGAL, low group with additional lymph node retrieval; CEA, carcinoembryonic antigen; ASA PS, American Society of Anesthesiologists physical status; WD, well differentiated; MD, moderate differentiated; PD, poorly differentiated; $\mathrm{MU}$, mucinous; SRC, signet ring cell.

Age, preoperative CEA and operation time were analyzed using the Student's ttest. The others were analyzed by chi-square test.

fect (demonstrated using a contrast study or computed tomography $[\mathrm{CT}])$ in the intestinal wall integrity at the anastomotic site regardless of clinical symptoms. Intestinal obstruction was diagnosed based on signs, symptoms, and radiographic evidence of small bowel obstruction or in those with evidence of impairment in gastrointestinal motility. All patients were followed-up with clinical examination, assessment of tumor markers, colonoscopy, and abdominopelvic and chest CT. All patients underwent a follow-up study every 3 months for the first 2 years and every 6 months thereafter for the next 3 years. If necessary, positron emission tomography was performed to assess recurrence or metastases. Disease-free survival (DFS) was defined as the period without disease including relapse, recurrence, or a second primary cancer including non-colorectal cancers and death due to any cause [12].

\section{Statistics}

All statistical analyses were performed using IBM SPSS Statistics ver. 22.0 (IBM Co., Armonk, NY, USA) and R ver. 3.5 (http://web-r. org). Clinicopathological characteristics, postoperative complications, and recurrence were compared using the Pearson chi-square test or Fisher exact test. Operation time, age, and harvested LN were analyzed using Student $\mathrm{t}$-test. DFS and overall survival (OS) rates were analyzed using Kaplan-Meier survival analysis with the logrank test. P-values $<0.05$ were considered statistically significant.

\section{RESULTS}

The characteristics of the 194 patients are shown in Table 1. There were no significant differences between the 2 groups. The mean follow-up time was $42.6 \pm 18.6$ months in $\mathrm{HG}$ and $37.2 \pm 21.1$ months in LGAL $(\mathrm{P}=0.063)$.

Results of harvested LN are shown in Table 2. The pathologic node stage was not different between the 2 groups $(\mathrm{P}=0.495)$. 
Table 2. Pathologic results of LNs

\begin{tabular}{lccc}
\hline Pathologic result & $\mathrm{HG}(\mathrm{n}=97)$ & LGAL $(\mathrm{n}=97)$ & P-value \\
\hline N stage & & & 0.495 \\
0 & $56(57.7)$ & $56(57.7)$ & \\
1a & $18(18.6)$ & $17(17.5)$ & \\
1b & $14(14.4)$ & $12(12.4)$ & \\
1c & $0(0)$ & $1(1.0)$ & \\
2a & $4(4.1)$ & $9(9.3)$ & \\
2b & $5(5.2)$ & $2(2.1)$ & \\
Total harvested LNs & $20.9 \pm 13.2$ & $20.2 \pm 9.2$ & 0.673 \\
Total metastatic LNs & $1.75(0-30)$ & $1.15(0-12)$ & 0.247 \\
Total harvested LNs of IMA & - & $2.19(0-11)$ & - \\
Total metastatic LNs of IMA & - & $0.01(0-1)$ & - \\
\hline
\end{tabular}

Values are presented as number (\%), mean \pm standard deviation, or mean (range). HG, high group; LGAL, low group with additional LN retrieval; LN, lymph node; IMA, inferior mesenteric artery.

$\mathrm{N}$ stage was analyzed by chi-square test. Total harvested LNs and total metastatic LNs were analyzed using the Student's t-test.

Table 3. Morbidity between the 2 groups

\begin{tabular}{lccc}
\hline Morbidity & HG $(\mathrm{n}=97)$ & LGAL $(\mathrm{n}=97)$ & P-value \\
\hline $\begin{array}{l}\text { Overall morbidity } \\
\text { No }\end{array}$ & $75(77.3)$ & $67(69.1)$ & 0.257 \\
Yes & $22(22.7)$ & $30(30.9)$ & \\
Anastomotic leakage & & & 0.030 \\
$\quad$ No & $83(85.6)$ & $92(94.8)$ & \\
Yes & $14(14.4)$ & $5(5.2)$ & \\
Wound & & & 0.213 \\
No & $96(99.0)$ & $92(94.8)$ & \\
Yes & $1(1.0)$ & $5(5.2)$ & \\
Infection & & & \\
No & $96(99.0)$ & $90(92.8)$ & \\
Yes & $1(1.0)$ & $7(7.2)$ & \\
Obstruction & & & \\
No & $95(97.9)$ & $85(87.6)$ & \\
Yes & $2(2.1)$ & $12(12.4)$ & \\
Others & & & \\
No & $96(99.0)$ & $95(97.9)$ & \\
Yes & $1(1.0)$ & $2(2.1)$ & \\
\hline
\end{tabular}

Values are presented as number (\%).

$H G$, high group; LGAL, low group with additional lymph node retrieval.

All values were analyzed by chi-square test.

The mean number of the total harvested LNs in all patients was $20.6 \pm 11.4$. The mean number of total harvested LN was $20.9 \pm$ 13.2 in the HG and 20.2 \pm 9.2 in the LGAL $(\mathrm{P}=0.673)$. The mean number of total metastatic LNs was 1.45 (range, 0-30) in total patients, 1.75 (range, 0-30) in the HG, and 1.15 (range, 0-12) in the
Table 4. Recurrence between the 2 groups

\begin{tabular}{lccc}
\hline Recurrence & $\mathrm{HG}(\mathrm{n}=97)$ & LGAL $(\mathrm{n}=97)$ & P-value \\
\hline Total recurrence & & & 0.122 \\
$\quad$ No & $85(87.6)$ & $77(79.4)$ & \\
$\quad$ Yes & $12(12.4)$ & $20(20.6)$ & \\
Systemic metastasis & & & 0.159 \\
No & $86(88.7)$ & $79(81.4)$ & \\
Yes & $11(11.3)$ & $18(18.6)$ & \\
Local recurrence & & & 0.621 \\
No & $96(99.0)$ & $94(96.9)$ & \\
Yes & $1(1.0)$ & $3(3.1)$ & \\
\hline
\end{tabular}

Values are presented as number (\%).

HG, high group; LGAL, low group with additional lymph node retrieval. All values were analyzed by chi-square test.

LGAL $(P=0.247)$. The mean number of additional harvested LN was 2.19 (range, $0-11$ ) in the LGAL, and one patient had a metastatic LN among the additional harvested LN in this group.

The morbidity rates in the groups are shown in Table 3 . The overall morbidity was $22.7 \%$ in the HG and $30.9 \%$ in the LGAL (P $=0.257)$. Anastomotic leakage occurred in 14 patients $(14.4 \%)$ in the $\mathrm{HG}$ and 5 patients $(5.2 \%)$ in the LGAL $(\mathrm{P}=0.030)$. Obstruction and ileus were more common in the LGAL than in the HG (P $=0.013$ ) and occurred in 2 patients $(2.1 \%)$ in the HG and 12 patients $(12.4 \%)$ in the LGAL. Most patients with obstruction were managed conservatively including nasogastric tube insertion and nothing pre ora (NPO). However, 1 patient in the LGAL underwent reoperation due to intestinal obstruction. Overall, 8 patients developed infection including pneumonia, catheter infection, or Clostridium colitis.

Of the 194 patients studied, 32 (16.5\%) showed metastases (Table 4). Recurrence was noted in 12 patients (12.4\%) in the HG and 20 patients $(20.6 \%)$ in the LGAL $(P=0.122)$. Systemic metastases occurred in 11 patients $(11.3 \%)$ in the $\mathrm{HG}$ and 18 patients $(18.6 \%)$ in the LGAL $(\mathrm{P}=0.159)$. In the HG patients with metastases, 2 had only liver metastasis, 5 had only lung metastasis, 1 had liver and lung metastases, and 4 had metastasis to other organs. In the LGAL patients with metastases, 4 had only liver metastasis, 8 had only lung metastasis, 1 had liver and lung metastases, and 7 had metastasis to other organs.

The overall mean DFS time in all participants was $53.9 \pm 1.8$ months. The mean DFS time was $57.7 \pm 2.1$ months in the HG and $45.9 \pm 2.4$ months in the LGAL $(\mathrm{P}=0.008)$ (Fig. 1). The OS rates are shown in Fig. 2. The mean OS time in all participants was $70.4 \pm 1.3$ months. The mean OS time was $63.7 \pm 1.6$ months in the HG and 69.1 \pm 2.6 months in the LGAL $(\mathrm{P}=0.386)$ (Fig. 2).

\section{DISCUSSION}

This study showed that the mean number of LNs in the LGAL 

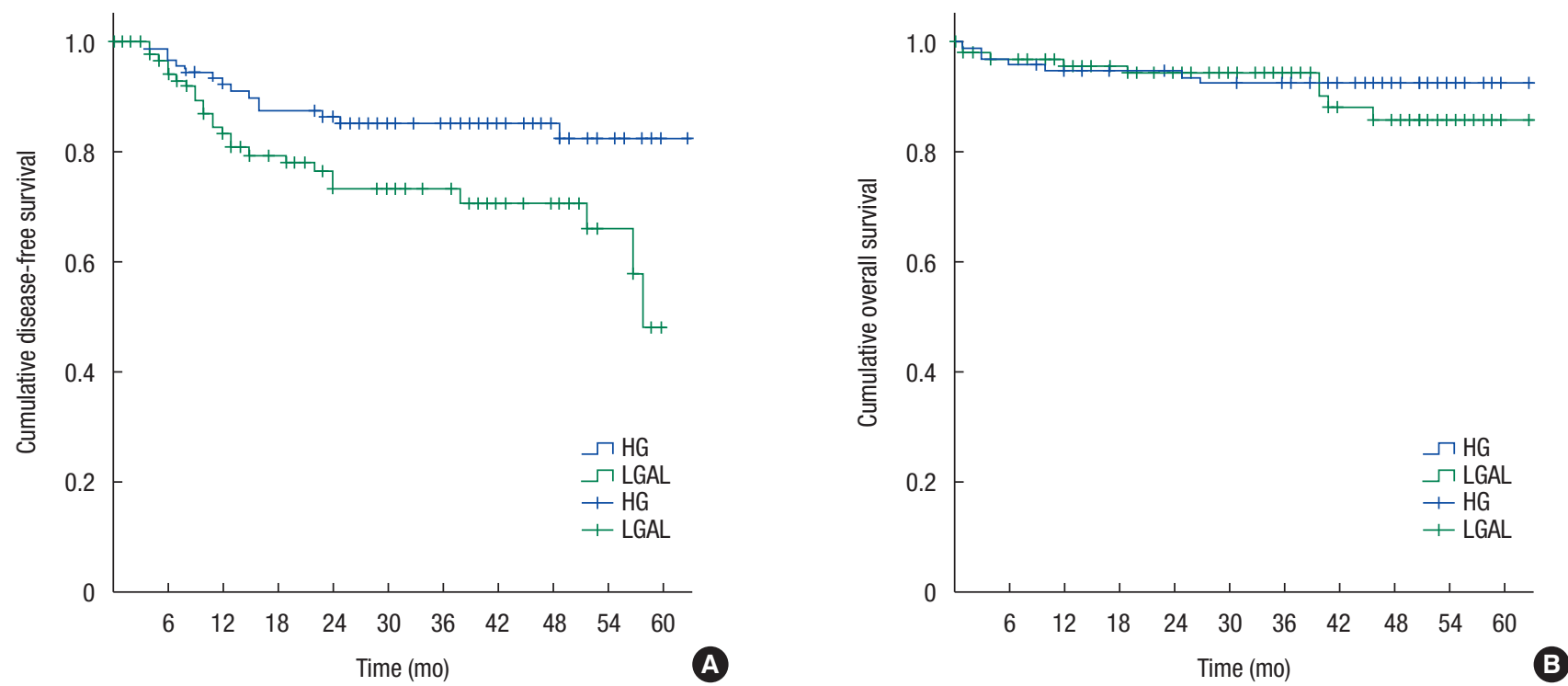

Fig. 2. Disease-free survival and overall survival between the 2 groups. $(\mathrm{A})$ Disease-free survival $(\mathrm{P}=0.008)$, $(\mathrm{B})$ overall survival $(\mathrm{P}=0.386)$. HG, high group; LGAL, low group with additional lymph node retrieval.

was not lower than that in the HG. One patient in the LGAL had a metastatic LN among the additional LN retrieved. Postoperatively, there were no significant differences in morbidity between the 2 groups. However, anastomotic leakage was more frequent in the HG than in the LGAL. No significant intergroup difference was observed in recurrence or OS rate. However, the DFS in the LGAL was lower than that in the HG.

An adequate number of LNs need to be retrieved for curative treatment of colorectal cancer [13]. Several studies have reported that inadequate LN retrieval was related to poor survival in patients with colorectal cancer $[14,15]$ and have recommended that at least 12 LNs be retrieved from a colorectal specimen [14-16]. Both groups in this study had retrieval $>12$ LNs. Previous studies have reported that the mean number of harvested LNs between the origin of the IMA and the root of the left colic artery was approximately 2-3 [3], and the mean number of metastatic LNs between the origin of the IMA and the root of the left colic artery was approximately 1.9-3 $[3,17]$. The tumor invasion rate of apical LNs was reported to be $1.7 \%-5.8 \%[3,18]$. In this study, the number of total harvested LN at the origin of the IMA concurred with other studies, and the tumor invasion rate of apical LN was 1.0\%.

The relationship between level of ligation of the IMA and morbidity rate remains controversial. Most studies have shown similar overall complication rates between patients undergoing high and low ligation of the IMA $[17,19]$. Reportedly, overall morbidity rates were approximately $19 \%-28 \%$ in the high and $17 \%-30 \%$ in the low ligation groups $[17,19]$. In this study, the overall morbidity rate was $22 \%-30 \%$. However, morbidity rates vary based on the kinds of complications. A few studies have demonstrated that high ligation was related to more frequent anastomotic leakage
[20-22]. In one study, the anastomotic leakage rate was over $13 \%$ in patients undergoing left colic artery non-preservation surgery for rectal cancer [20]. A few other studies have shown similar rates of anastomotic leakage between patients undergoing high and low ligation of the IMA $[4,19]$. Anastomotic leakage was approximately $7 \%-10 \%$ for all cases of IMA ligation $[4,19]$. The overall anastomotic leakage rate was $9.3 \%$ in this study, and anastomotic leakage in the $\mathrm{HG}$ was significantly higher than that in the LGAL. We hypothesized that low ligation might affect anastomosis. Previous studies have shown that low ligation of the IMA is associated with better preservation of genitourinary function [19]. However, ileus and defecation were not improved in those undergoing low ligation of the IMA [23]. In this study, obstruction was more frequent in the LGAL than the HG. All but one patient was able to be managed conservatively. We suspect that low ligation of the IMA with additional LN retrieval resulted in a larger area without peritoneal coverage near the origin of the IMA, which may have adversely affected gastrointestinal motility.

The relationship between level of IMA ligation and survival remains controversial. High ligation of the IMA aids in LN dissection at its origin. Long-term survival in patients with metastatic $\mathrm{LN}$ at the origin of the IMA was poorer than that in patients without metastatic LN at that location, although other studies found that survival rates did not significantly differ based on level of IMA ligation [1, 17, 24]. Previous studies reported that the fiveyear OS rate is $82.4 \%-91.3 \%$ and $80.3 \%-90.2 \%$ in the high and low ligation groups, respectively $[17,24]$, in which the DFS rate was $75.6 \%-83.2 \%$ and $76.2 \%-78.0 \%$. In this study, the OS rate did not differ between groups, and the DFS rate in the LGAL was lower than that in the HG. 
Limitations of this study are that it was a non-randomized trial and selection biases cannot be ignored. We used case-control matching to overcome the drawbacks associated with the retrospective study design. The follow-up period was longer in the HG than that in the LGAL, although this difference was not statistically significant. High ligation is a conventional procedure that has been performed for a long time in clinical settings. Low ligation with additional LN retrieval is a relatively new technique, and this may be the reason for the shorter follow-up in this group.

No significant intergroup differences were observed in OS or recurrence rate. Anastomotic leakage and DFS were lower in the LGAL. Low ligation of the IMA with additional LN retrieval might be a safe procedure in the surgical management of patients with colorectal cancer. However, oncologic benefits were greater with high ligation of the IMA. Sufficiently powered studies are warranted in the future to provide a better understanding of the differences in clinical outcomes associated with these procedures.

\section{CONFLICT OF INTEREST}

No potential conflict of interest relevant to this article was reported.

\section{REFERENCES}

1. Pezim ME, Nicholls RJ. Survival after high or low ligation of the inferior mesenteric artery during curative surgery for rectal cancer. Ann Surg 1984;200:729-33.

2. Lange MM, Buunen M, van de Velde CJ, Lange JF. Level of arterial ligation in rectal cancer surgery: low tie preferred over high tie. A review. Dis Colon Rectum 2008;51:1139-45.

3. Kanemitsu Y, Hirai T, Komori K, Kato T. Survival benefit of high ligation of the inferior mesenteric artery in sigmoid colon or rectal cancer surgery. Br J Surg 2006;93:609-15.

4. Cirocchi R, Trastulli S, Farinella E, Desiderio J, Vettoretto N, Parisi A, et al. High tie versus low tie of the inferior mesenteric artery in colorectal cancer: a RCT is needed. Surg Oncol 2012;21:e111-23.

5. Bonnet S, Berger A, Hentati N, Abid B, Chevallier JM, Wind P, et al. High tie versus low tie vascular ligation of the inferior mesenteric artery in colorectal cancer surgery: impact on the gain in colon length and implications on the feasibility of anastomoses. Dis Colon Rectum 2012;55:515-21.

6. Thum-umnuaysuk S, Boonyapibal A, Geng YY, Pattana-Arun J. Lengthening of the colon for low rectal anastomosis in a cadaveric study: how much can we gain? Tech Coloproctol 2013;17:37781.

7. Uehara K, Yamamoto S, Fujita S, Akasu T, Moriya Y. Impact of upward lymph node dissection on survival rates in advanced lower rectal carcinoma. Dig Surg 2007;24:375-81.

8. Seike K, Koda K, Saito N, Oda K, Kosugi C, Shimizu K, et al. Laser Doppler assessment of the influence of division at the root of the inferior mesenteric artery on anastomotic blood flow in rec- tosigmoid cancer surgery. Int J Colorectal Dis 2007;22:689-97.

9. Kawamura YJ, Umetani N, Sunami E, Watanabe T, Masaki T, Muto T. Effect of high ligation on the long-term result of patients with operable colon cancer, particularly those with limited nodal involvement. Eur J Surg 2000;166:803-7.

10. Komen N, Slieker J, de Kort P, de Wilt JH, van der Harst E, Coene $\mathrm{PP}$, et al. High tie versus low tie in rectal surgery: comparison of anastomotic perfusion. Int J Colorectal Dis 2011;26:1075-8.

11. Lemmens VE, Janssen-Heijnen ML, Verheij CD, Houterman S, Repelaer van Driel OJ, Coebergh JW. Co-morbidity leads to altered treatment and worse survival of elderly patients with colorectal cancer. Br J Surg 2005;92:615-23.

12. Chua YJ, Sargent D, Cunningham D. Definition of disease-free survival: this is my truth-show me yours. Ann Oncol 2005;16: 1719-21.

13. Chang GJ, Rodriguez-Bigas MA, Skibber JM, Moyer VA. Lymph node evaluation and survival after curative resection of colon cancer: systematic review. J Natl Cancer Inst 2007;99:433-41.

14. Swanson RS, Compton CC, Stewart AK, Bland KI. The prognosis of T3N0 colon cancer is dependent on the number of lymph nodes examined. Ann Surg Oncol 2003;10:65-71.

15. Chen HH, Chakravarty KD, Wang JY, Changchien CR, Tang R. Pathological examination of 12 regional lymph nodes and longterm survival in stages I-III colon cancer patients: an analysis of 2,056 consecutive patients in two branches of same institution. Int J Colorectal Dis 2010;25:1333-41.

16. Le Voyer TE, Sigurdson ER, Hanlon AL, Mayer RJ, Macdonald JS, Catalano PJ, et al. Colon cancer survival is associated with increasing number of lymph nodes analyzed: a secondary survey of intergroup trial INT-0089. J Clin Oncol 2003;21:2912-9.

17. Yasuda K, Kawai K, Ishihara S, Murono K, Otani K, Nishikawa T, et al. Level of arterial ligation in sigmoid colon and rectal cancer surgery. World J Surg Oncol 2016;14:99.

18. Alici A, Kement M, Gezen C, Akin T, Vural S, Okkabaz N, et al. Apical lymph nodes at the root of the inferior mesenteric artery in distal colorectal cancer: an analysis of the risk of tumor involvement and the impact of high ligation on anastomotic integrity. Tech Coloproctol 2010;14:1-8.

19. Mari GM, Crippa J, Cocozza E, Berselli M, Livraghi L, Carzaniga P, et al. Low ligation of inferior mesenteric artery in laparoscopic anterior resection for rectal cancer reduces genitourinary dysfunction: results from a randomized controlled trial (HIGHLOW trial). Ann Surg 2018 Jul 10 [Epub]. http://dx.doi.org/10.1097/ SLA.0000000000002947.

20. Hinoi T, Okajima M, Shimomura M, Egi H, Ohdan H, Konishi F, et al. Effect of left colonic artery preservation on anastomotic leakage in laparoscopic anterior resection for middle and low rectal cancer. World J Surg 2013;37:2935-43.

21. Zeng J, Su G. High ligation of the inferior mesenteric artery during sigmoid colon and rectal cancer surgery increases the risk of anastomotic leakage: a meta-analysis. World J Surg Oncol 2018; $16: 157$. 
22. Shiomi A, Ito M, Maeda K, Kinugasa Y, Ota M, Yamaue H, et al. Effects of a diverting stoma on symptomatic anastomotic leakage after low anterior resection for rectal cancer: a propensity score matching analysis of 1,014 consecutive patients. J Am Coll Surg 2015;220:186-94.

23. Matsuda K, Hotta T, Takifuji K, Yokoyama S, Oku Y, Watanabe T, et al. Randomized clinical trial of defaecatory function after ante- rior resection for rectal cancer with high versus low ligation of the inferior mesenteric artery. Br J Surg 2015;102:501-8.

24. Fujii S, Ishibe A, Ota M, Suwa H, Watanabe J, Kunisaki C, et al. Short-term and long-term results of a randomized study comparing high tie and low tie inferior mesenteric artery ligation in laparoscopic rectal anterior resection: subanalysis of the HTLT (high tie vs. low tie) study. Surg Endosc 2019;33:1100-10. 